\title{
In-situ, Ex-situ, and 3-D Imaging of Nanomaterials in the STEM
}

Ilke Arslan

Physical Sciences Division, Pacific Northwest National Laboratory, Richland, WA, USA

Many processes in materials science are dependent on the local environmental conditions (temperature, pressure, gas, liquid etc). Although recent years have seen a paradigm change in (scanning) transmission electron microscopy ((S)TEM) with unprecedented improvements in spatial and spectroscopic resolution being achieved, a full utilization of these new capabilities to study processes requires precise control of the environment around the sample. Further, as nanomaterials are three-dimensional (3-D) in nature, it is not sufficient to take single 2-D projection images of 3-D objects. In order to understand the true nature of the nanomaterial, a 3$\mathrm{D}$ tomogram is necessary on the nano- to atomic scale. Traditionally, this involves taking a series of images of the sample at different tilt angles, normally ranging between $-70^{\circ}$ to $+70^{\circ}$ every 1 to 2 degrees, and using these two dimensional images to reconstruct a three dimensional volume of the sample. More recently, the development of novel algorithms have greatly aided in the reduction of reconstruction artifacts, and even provided atomic resolution 3-D tomograms from only 3-5 projection images.

We have recently developed new algorithms for post-acquisition processing for electron tomography data. Using a combination of compressed sensing and discrete reconstruction methods, one of the algorithms not only provides accurate reconstructions with limited projection images, but also with a limited tilt range. This algorithm offers a solution to the decades-old "missing wedge" artifact present in reconstructions with limited data. With the ability to provide high quality reconstructions with only $\sim 30$ images as opposed to the standard $\sim 120$, this algorithm opens the door to materials characterization of electron beam sensitive materials across all fields of science (Figures 1 and 2). The reduced number of images necessary for a robust 3-D reconstruction also opens the door for 3-D chemical imaging, using EDX or EELS, where the number of tilt 'images' are also limited.

Furthermore, the reduced number of images necessary for reconstructions moves us closer to combining 3-D imaging with in-situ/ex-situ methods to get closer to the conditions in which the material will be used, such as high temperature, liquid environments, gas environments or a combination thereof. Combining an in-situ or ex-situ experiment with STEM tomography is a very powerful method for materials characterization. The benefits and limitations of all these methods will be discussed through examples of different inorganic nanomaterials.

This research was supported in part by the LDRD program at the Pacific Northwest National Laboratory (PNNL). PNNL is operated by Battelle for the DOE under contract DE-AC0576RL01830. 

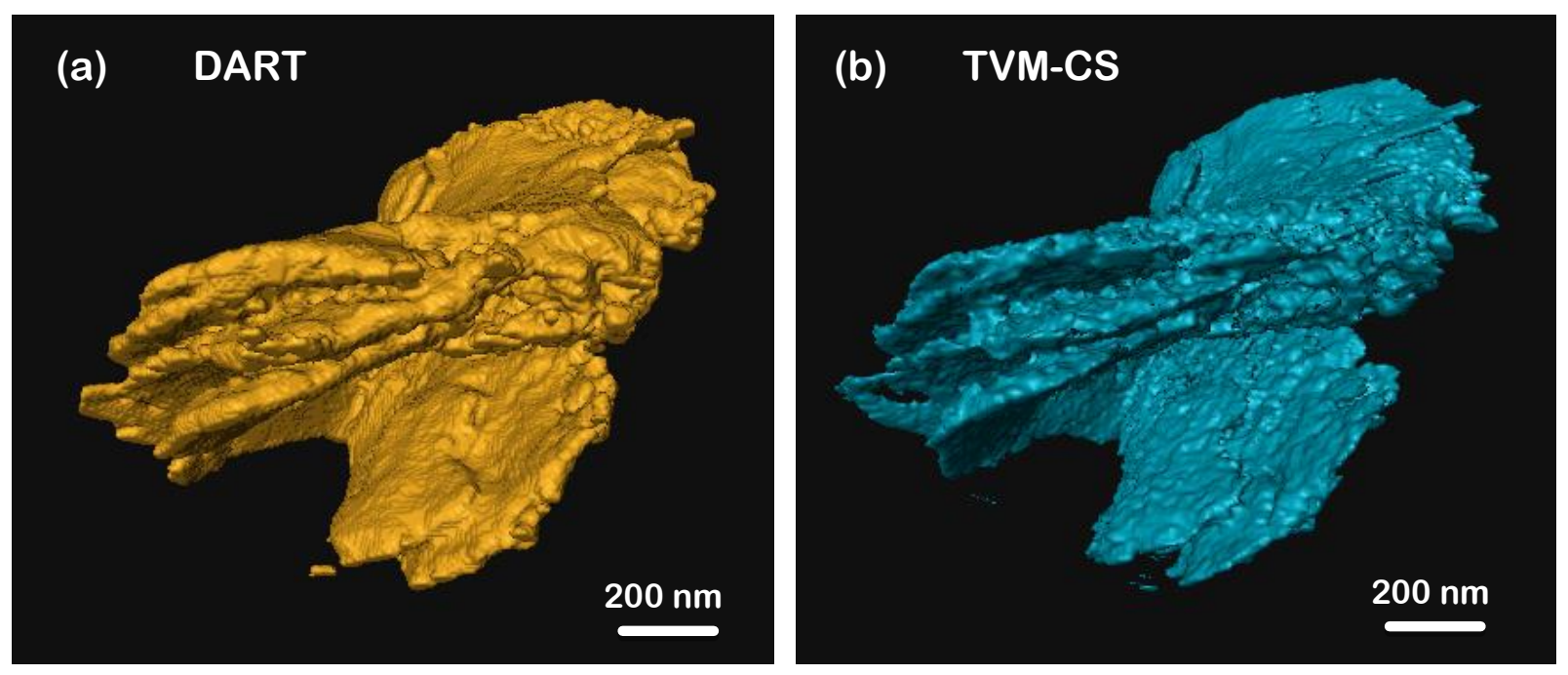

Figure 1. (a) A layered zeolite reconstructed using DART, and (b) the same volume reconstructed using TV minimization within CS.
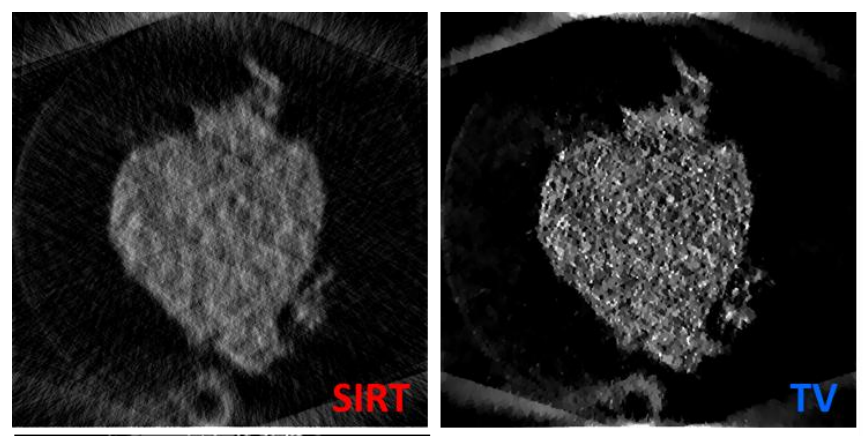

\section{Projections}
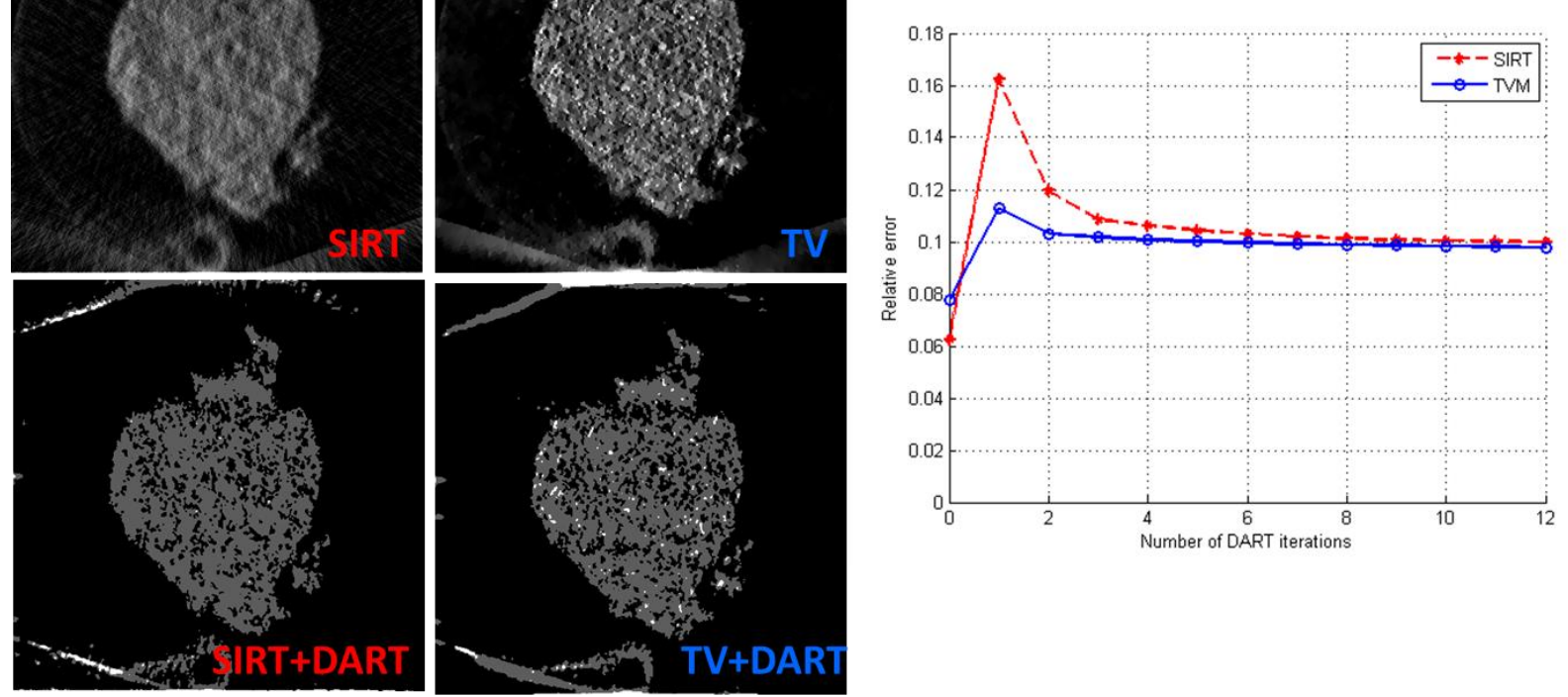

Figure 2. A comparison of DART reconstructions using SIRT and TV as initial solutions using half of the projections in a data set, in this case 37 images. The top images show the initial solutions, while the bottom images show slices through the final reconstruction. The graph displays the relative error using each method. 